\title{
Dengue Virus
}

National Cancer Institute

\section{Source}

National Cancer Institute. Dengue Virus. NCI Thesaurus. Code C112036.

A species of enveloped and spherical viruses with a capsid with $\mathrm{T}=3$ icosahedral symmetry in the Flaviviridae family and Flavivirus genus. The genome is composed of non-segmented, single-stranded, positive-sense RNA. Dengue viruses are found in nonhuman primates and humans and cause dengue fever in humans. Dengue viruses are transmitted mainly via the bite of infected mosquitoes of the genus Aedes. 